\title{
2-Cys peroxiredoxin is required in successful blood-feeding, reproduction, and antioxidant response in the hard tick Haemaphysalis longicornis
}

Kodai Kusakisako ${ }^{1,2}$, Remil Linggatong Galay ${ }^{3}$, Rika Umemiya-Shirafuji ${ }^{4}$, Emmanuel Pacia Hernandez ${ }^{1,2}$, Hiroki Maeda ${ }^{1,2}$, Melbourne Rio Talactac ${ }^{1,2}$, Naotoshi Tsuji ${ }^{5}$, Masami Mochizuki ${ }^{1}$, Kozo Fujisaki ${ }^{6}$ and Tetsuya Tanaka ${ }^{1,2^{*}}$

\begin{abstract}
Background: Ticks are obligate hematophagous arthropods that feed on vertebrate blood that contains iron. Ticks also concentrate host blood with iron; this concentration of the blood leads to high levels of iron in ticks. The hostderived iron reacts with oxygen in the tick body and this may generate high levels of reactive oxygen species, including hydrogen peroxide $\left(\mathrm{H}_{2} \mathrm{O}_{2}\right)$. High levels of $\mathrm{H}_{2} \mathrm{O}_{2}$ cause oxidative stress in organisms and therefore, antioxidant responses are necessary to regulate $\mathrm{H}_{2} \mathrm{O}_{2}$. Here, we focused on peroxiredoxin ( $\mathrm{Prx}$ ), an $\mathrm{H}_{2} \mathrm{O}_{2}$-scavenging enzyme in the hard tick Haemaphysalis longicornis.

Methods: The mRNA and protein expression profiles of 2-Cys peroxiredoxin (HIPrx2) in H. longicornis were investigated in whole ticks and internal organs, and developmental stages, using real-time PCR and Western blot analysis during blood-feeding. The localization of HIPrx2 proteins in tick tissues was also observed by immunostaining. Moreover, knockdown experiments of HIPrx2 were performed using RNA interference to evaluate its function in ticks.

Results: Real-time PCR showed that HIPrx2 gene expression in whole ticks and internal organs was significantly upregulated by blood-feeding. However, protein expression, except in the midgut, was constant throughout bloodfeeding. Knockdown of the HIPrx2 gene caused significant differences in the engorged body weight, egg weight and hatching rate for larvae as compared to the control group. Finally, detection of $\mathrm{H}_{2} \mathrm{O}_{2}$ after knockdown of HIPrxs in ticks showed that the concentration of $\mathrm{H}_{2} \mathrm{O}_{2}$ significantly increased before and after blood-feeding.

Conclusion: Therefore, HIPrx2 can be considered important for successful blood-feeding and reproduction through the regulation of $\mathrm{H}_{2} \mathrm{O}_{2}$ concentrations in ticks before and after blood-feeding. This study contributes to the search for a candidate target for tick control and further understanding of the tick's oxidative stress coping mechanism during blood-feeding.
\end{abstract}

Keywords: Peroxiredoxin, Haemaphysalis longicornis, RNA interference, Hydrogen peroxide

(Continued on next page)

\footnotetext{
* Correspondence: tetsuya@ms.kagoshima-u.ac.jp

1 Laboratory of Infectious Diseases, Joint Faculty of Veterinary Medicine,

Kagoshima University, Korimoto, Kagoshima 890-0065, Japan

${ }^{2}$ Department of Pathological and Preventive Veterinary Science, United

Graduate School of Veterinary Science, Yamaguchi University, Yoshida,

Yamaguchi 753-8515, Japan

Full list of author information is available at the end of the article
}

(c) 2016 The Author(s). Open Access This article is distributed under the terms of the Creative Commons Attribution 4.0 International License (http://creativecommons.org/licenses/by/4.0/), which permits unrestricted use, distribution, and reproduction in any medium, provided you give appropriate credit to the original author(s) and the source, provide a link to the Creative Commons license, and indicate if changes were made. The Creative Commons Public Domain Dedication waiver (http://creativecommons.org/publicdomain/zero/1.0/) applies to the data made available in this article, unless otherwise stated. 


\begin{abstract}
(Continued from previous page)
Abbreviations: $\mathrm{H}_{2} \mathrm{O}_{2}$, Hydrogen peroxide; Prx, Peroxiredoxin; HIPrx2, Haemaphysalis longicornis 2-Cys peroxiredoxin; ROS, Reactive oxygen species; SDS-PAGE, Sodium dodecyl sulfate-polyacrylamide gel electrophoresis; HIPrx, Haemaphysalis longicornis 1-Cys peroxiredoxin; PBS, Phosphate buffered saline; IFAT, Indirect immunofluorescent antibody test; RNAi, RNA interference; dsHIPrx2, The double-stranded RNA of HIPrxz; dsHIPrx, The double-stranded RNA of HIPrx; dsLuc, The double-stranded RNA of the firefly luciferase; dsDouble, The doublestranded RNA mixture of dsHIPrx and dsHIPrx2 at $1 \mu \mathrm{g}$ concentration each; SA, The acinar cells of the salivary glands; SGG, The granular cells of the salivary glands; SD, The salivary duct
\end{abstract}

\section{Background}

In high concentrations, hydrogen peroxide $\left(\mathrm{H}_{2} \mathrm{O}_{2}\right)$ is known to be a harmful chemical compound to aerobic organisms due to its ability to seriously damage membrane lipids, nucleic acids, and proteins [1]. Almost all aerobic organisms have developed defense systems to scavenge $\mathrm{H}_{2} \mathrm{O}_{2}$. Catalases, peroxidases, and peroxiredoxins (Prxs) are scavengers of $\mathrm{H}_{2} \mathrm{O}_{2}$ [2]. Prxs are ubiquitous antioxidant enzymes investigated in various organisms [3]. Particularly, high levels of Prxs are produced in mammalian cells, including erythrocytes [4]. Erythrocytes are exposed to more oxidative stress than any other cell type, due to the abundance of heme iron and oxygen, which can generate $\mathrm{H}_{2} \mathrm{O}_{2}$ [5]. These indicate that Prxs may have important roles in peroxide detoxification in cells.

Prxs can be divided into two groups according to the presence of one or two highly conserved cysteines in organisms, 1-Cys or 2-Cys Prx [3]. 2-Cys Prxs are identified by two conserved cysteines, peroxidatic and resolving cysteines [6]. On the other hand, the 1-Cys Prxs have a conserved peroxidatic cysteine and do not contain a resolving cysteine [7]. Enzymes of the Prx family exhibit antioxidant activity that catalyzes the reduction of $\mathrm{H}_{2} \mathrm{O}_{2}$ into water $\left(\mathrm{H}_{2} \mathrm{O}\right)$, with thioredoxin as an immediate hydrogen donor or donor thiol, respectively [7].

In some endoparasites, such as Plasmodium and Fasciola parasites, Prxs have been characterized as antigens or secreted proteins, suggesting that endoparasite Prxs may participate in interactions between the parasites and their hosts $[8,9]$. Therefore, to evaluate the efficacy of antigens for these endoparasites, basic biological and bio-histological analyses such as mRNA and protein expression profiles, and the localization of proteins in these parasites have been studied.

Ticks need blood meals to develop from one stage to the next and for reproduction. Blood-feeding and the digestion of blood provide nutrition and energy for molting, development, and the vitellogenesis of ticks [10]. Ticks feed on vertebrate blood that contains iron, such as heme, ferrous iron, and other pro-oxidants. Ticks also concentrate host blood with iron; this concentration of the blood leads to high levels of iron in ticks. Host- derived iron may react with oxygen in the tick body, and then high levels of reactive oxygen species (ROS), including $\mathrm{H}_{2} \mathrm{O}_{2}$, may be generated [11]. Haemaphysalis longicornis 1-Cys Prx (HlPrx) has been reported previously; however, there is still little knowledge about the biological functions of Prxs in ticks [12].

In the present study, we analyzed mRNA and protein expression profiles and the localization of proteins in tick tissues of $H$. longicornis 2-Cys Prx, HlPrx2, identified previously [13]. Moreover, HlPrx and/or HlPrx2 gene silencing was performed to clarify their functions in ticks using RNA interference. Finally, we demonstrated that the double knockdown of HlPrx and HlPrx2 led to increased oxidative stress in ticks.

\section{Methods}

\section{Ticks and animals}

The parthenogenetic Okayama strain of $H$. longicornis has been maintained by blood-feeding on the ears of Japanese white rabbits (KBT Oriental Co. Ltd, Saga, Japan) in the Laboratory of Infectious Diseases, Joint Faculty of Veterinary Medicine, Kagoshima University [14]. Rabbits were cared for in accordance with the guidelines approved by the Animal Care and Use Committee of Kagoshima University (Approval no. VM13007) and maintained under regulated conditions throughout the experiments.

\section{Total RNA extraction and CDNA synthesis}

To extract total RNA, whole ticks were homogenized using an Automill (Tokken, Chiba, Japan), while dissected organs were disrupted using a pellet pestle motor (SigmaAldrich, St. Louis, MO, USA). The extracted RNA was purified using TRI Reagent (Sigma-Aldrich), and then treated with an RQ1 RNase-Free DNase (Promega, Madison, WI, USA). cDNA synthesis was performed with ReverTra Ace- $\alpha$ - (Toyobo, Osaka, Japan) following the manufacturer's protocol using $1 \mu \mathrm{g}$ of total RNA.

\section{Expression analysis of HIPrx2 mRNA}

The expression analysis of the HlPrx 2 mRNA was performed with real-time PCR using THUNDERBIRD 
SYBR qPCR Mix (Toyobo) with a 7300 real-time PCR system (Applied Biosystems, Foster City, CA, USA). Gene-specific primers were designed to target HlPrx2 and the internal control genes, as shown in Table 1 . Standard curves were made from four-fold serial dilutions of the cDNA of adult ticks fed for three days. The PCR cycle profile was as follows: initial denaturation at $95{ }^{\circ} \mathrm{C}$ for $10 \mathrm{~min}, 40$ cycles of a denaturation step at $95^{\circ} \mathrm{C}$ for $15 \mathrm{~s}$, and an annealing/extension step at $60^{\circ} \mathrm{C}$ for $60 \mathrm{~s}$. The data was analyzed with 7300 system SDS software (Applied Biosystems). At the first step of real-time PCR, actin, tubulin, $P O$, and $L 23$ genes were evaluated for standardization and $L 23$ was selected as the tick reference in the current study.

\section{Production of an antiserum against recombinant HIPrx2}

To prepare mouse anti-HlPrx2 sera, $100 \mu \mathrm{g}$ of recombinant HIPrx2 (rHIPrx2; [13]) was completely mixed with Freund's complete adjuvant (Sigma-Aldrich) and intraperitoneally injected into ddY female mice (four weeks old, Kyudo, Saga, Japan). After two weeks, these mice were injected with $100 \mu \mathrm{g}$ of rHlPrx2 with Freund's incomplete adjuvant (Sigma-Aldrich) twice at a twoweek interval to boost the generation of antibodies against rHIPrx2. Their blood was collected two weeks after the third immunization to obtain specific antisera to rHlPrx2.

\section{Protein extraction and Western blot analysis}

Homogenized ticks were suspended in phosphate buffered saline (PBS) and ultrasonicated three times, two minutes each (Vibra-Cell ${ }^{\mathrm{TM}}$; Sonics and Materials, Newtown, CT, USA) on ice and finally centrifuged at $500 \times g$. The supernatant was resolved in a $12 \%$ SDSPAGE gel under reducing conditions. After sodium dodecyl sulfate-polyacrylamide gel electrophoresis (SDSPAGE), the proteins were transferred onto a polyvinylidene difluoride membrane (Immobilon -P; Millipore, Danvers, MA, USA). The membrane was blocked overnight with $3 \%$ skim milk in PBS (pH 7.4) (blocking solution); it was incubated with a 1:500 dilution of antirHlPrx2 mouse sera in blocking solution at $37{ }^{\circ} \mathrm{C}$ for $1 \mathrm{~h}$. For loading control, tubulin was detected using antiserum against recombinant $H$. longicornis tubulin [15].

Table 1 Gene-specific primers used in this study

\begin{tabular}{|c|c|}
\hline Primer & Sequence $\left(5^{\prime}-3^{\prime}\right)$ \\
\hline HIPrx2 RT-F & TATGCCTAAGCTGGCGAAGC \\
\hline HIPrx2 RT-R & CAGGCGAGGTGAGAGAAGTG \\
\hline HIPrx RT-F & ATGAGGTCCTCCGTGCTACT \\
\hline HIPrx RT-R & TGCCACACCGTCATAAGCAT \\
\hline HIPrx2 real time-F & GTGTGCCCTGCTAACTGGAA \\
\hline HIPrx2 real time- $\mathrm{R}$ & ATGAGACACACGGGGCTTTG \\
\hline HIPrx2 T7-F & TAATACGACTCACTATAGG GATCAAGCTGTCCGATTACAAGAAC \\
\hline HIPrx2 T7-R & TAATACGACTCACTATAGG TTCCAGTTAGCAGGGCACACT \\
\hline HIPrx2 RNAi-F & GATCAAGCTGTCCGATTACAAGAAC \\
\hline HIPrx2 RNAi-R & TTCCAGTTAGCAGGGCACACT \\
\hline HIPrx T7-F & TAATACGACTCACTATAGG CACCACGGTTGGATCAAGGA \\
\hline HIPrx T7-R & TAATACGACTCACTATAGG TITGCAGAGCCACCACTCAA \\
\hline HIPrx RNAi-F & CACCACGGTTGGATCAAGGA \\
\hline HIPrx RNAi-R & TTTGCAGAGCCACCACTCAA \\
\hline Actin RT-F & CCAACAGGGAGAAGATGACG \\
\hline Actin RT-R & ACAGGTCCTTACGGATGTCC \\
\hline Actin real time-F & ATCCTGCGTCTCGACTTGG \\
\hline Actin real time- $\mathrm{R}$ & GCCGTGGTGGTGAAAGAGTAG \\
\hline Tubulin real time-F & TTCAGGGGCCGTATGAGTAT \\
\hline Tubulin real time- $R$ & TGTTGCAGACATCTTGAGGC \\
\hline $\mathrm{PO}$ real time- $\mathrm{F}$ & CTCCATTGTCAACGGTCTCA \\
\hline P0 real time- $R$ & TCAGCCTCCTTGAAGGTGAT \\
\hline L23 real time-F & CACACTCGTGTTCATCGTCC \\
\hline L23 real time- $\mathrm{R}$ & ATGAGTGTGTTCACGTTGGC \\
\hline
\end{tabular}

Italics denote T7 RNA polymerase promoter sequences 
After washing five times in PBS containing $0.05 \%$ Tween 20 (PBS-T), the membrane was incubated with a 1:50,000 dilution of horseradish peroxidase (HRP)-conjugated sheep anti-mouse IgG (Dako, Glostrup, Denmark) in blocking solution at $37{ }^{\circ} \mathrm{C}$ for $1 \mathrm{~h}$. After washing five times in PBS-T, bands were detected using Amersham $^{\mathrm{TM}} \mathrm{ECL}^{\mathrm{TM}}$ Prime Western Blotting Detection Reagent (GE Healthcare, Buckinghamshire, UK) and viewed using FluorChem FC2 software (Alpha Innotech, San Leandro, CA, USA). To accurately determine differences in the protein expression, band densitometry analysis was performed using Alpha View Software (Alpha Innotech). The band densitometry analysis results shown in this study represent the mean of three trials of Western blot analysis.

\section{Immunostaining}

To confirm the localization of HlPrx2 in tick tissues, indirect immunofluorescent antibody tests (IFAT) were performed. Engorged ticks were dissected under a stereomicroscope (SZX10, Olympus, Tokyo, Japan) for collecting tick internal organs. Dissected organs were fixed in a $4 \%$ paraformaldehyde phosphate buffer solution ( $\mathrm{pH}$ 7.4) that included $0.1 \%$ glutaraldehyde at $4{ }^{\circ} \mathrm{C}$ overnight. After washing with a sucrose series, organs were embedded in a Tissue-Tek O.C.T Compound (Sakura Finetek, Torrance, CA, USA). Frozen sections from each internal organ were cut to a thickness of $10 \mu \mathrm{m}$ using Kawamoto's film method (Leica Microsystems, Tokyo, Japan) and a cryostat (Leica CM 1850, Leica Microsystems, Wetzlar, Germany). The films were blocked with $5 \%$ skim milk in PBS (pH 7.4) (blocking solution) at $37^{\circ} \mathrm{C}$ for $1 \mathrm{~h}$, and then incubated with 1:50 dilution in a blocking solution of anti-HIPrx2 mouse serum at $37^{\circ} \mathrm{C}$ for $1 \mathrm{~h}$. For the negative control, normal mouse serum was used. After washing three times in PBS, the slides were incubated at $37{ }^{\circ} \mathrm{C}$ for $1 \mathrm{~h}$ with Alexa Fluor 594 goat anti-mouse IgG (Invitrogen, Carlsbad, CA, USA) with 1:1,000 dilution in the blocking solution. After removing the antibody by washing three times with PBS, the films were placed on a glass slide and mounted with DAPI (VECTASHIELD ; Vector Laboratories, Burlingame, CA, USA), and then covered with a cover glass. The images were recorded using a confocal laser scanning microscope (LSM700, Carl Zeiss, Jena, Germany). Hemocytes were prepared using a glass slide instead of film as described previously by Galay et al. [16]. Briefly, hemolymph collected from ticks by amputating the legs was smeared directly on glass slides and air-dried. After drying, hemocyte smears were fixed with $4 \%$ paraformaldehyde in PBS that included $0.1 \%$ glutaraldehyde. Thereafter, the same method used for the internal organs' IFAT was performed.

\section{RNA interference (RNAi)}

Two separate PCR reactions of approximately $469 \mathrm{bp}$ with a single T7 promoter were generated using the following primer sets: a T7-attached gene-specific forward primer (HIPrx2 T7-F) and gene-specific reverse primer (HlPrx2 RNAi-R) and a T7-attached gene-specific reverse primer (HlPrx2 T7-R) and gene-specific forward primer (HlPrx2 RNAi-F) (Table 1). After gel purification of PCR products using a GENECLEAN II KIT (MP Biomedicals, Irvine, CA, USA), double-stranded RNA of $H$. longicornis 2-Cys peroxiredoxin (dsHlPrx2) was synthesized using the T7 RiboMAX ${ }^{\mathrm{in}}$ Express RNAi System (Promega) with two separate single-promoter templates in accordance with the manufacturer's protocol. Doublestranded RNA of $H$. longicornis 1-Cys peroxiredoxin ( $d s H l P r x$ ) was also synthesized using $H l P r x$ gene-specific primers (Table 1). The firefly luciferase (Luc) gene [17] was used for control (dsLuc group). One microgram of $d s H l P r x$, dsHlPrx2, or dsDouble (dsHlPrx and dsHlPrx2 were mixed at $1 \mu \mathrm{g}$ concentration each) was injected into 30 unfed adult female ticks in each experimental group and $d s L u c$ group through the fourth coxae into the hemocoel. Injected ticks were observed for one day and subsequently transferred to rabbits with each group infesting separate ears. Three to four days after attachment, three ticks were manually detached to confirm gene silencing using RT-PCR. The remaining ticks were allowed to feed until engorgement, and the total number of engorged ticks, the engorged body weight, the oviposition, and the hatching rate were assessed.

\section{Detection of hydrogen peroxide $\left(\mathrm{H}_{2} \mathrm{O}_{2}\right)$ in adult female ticks during blood-feeding}

The $\mathrm{H}_{2} \mathrm{O}_{2}$ concentration in ticks was measured using the ferrous oxidation of xylenol orange assay [18]. Briefly, homogenized unfed and partially fed ticks were suspended in $200 \mu \mathrm{l}$ of Milli-Q $\mathrm{H}_{2} \mathrm{O}$, while homogenized engorged ticks were suspended in $900 \mu \mathrm{l}$ of Milli-Q $\mathrm{H}_{2} \mathrm{O}$. The samples were centrifuged at $500 \times g$, and the supernatant was collected. The supernatant from the engorged ticks was further diluted 10 times in Milli-Q $\mathrm{H}_{2} \mathrm{O}$. Ninety microliters of the supernatant from unfed and partially fed ticks or the diluted supernatant from engorged ticks was used for a sample solution as described later. The assay reagent consisted of $125 \mu \mathrm{M}$ xylenol orange, $250 \mu \mathrm{M}$ ammonium iron (II) sulfate, $100 \mathrm{mM}$ sorbitol, and $25 \mathrm{mM}$ sulfuric acid. One hundred microliters of the sample solutions was added to a $1-\mathrm{ml}$ assay reagent. The mixture was vortexed immediately, left at room temperature for $30 \mathrm{~min}$, and measured at $560 \mathrm{~nm}$ using a spectrophotometer (Ultrospec 2100 pro; GE Healthcare, Pittsburgh, PA, USA). Finally, the ratio of the $\mathrm{H}_{2} \mathrm{O}_{2}$ concentration $(\mu \mathrm{M})$ to the corresponding tick's body weight $(\mathrm{mg})$ was calculated. 


\section{Statistical analysis}

All experiments were conducted in two or three separate trials. Data except for hatching rate were statistically analyzed using Welch's $t$-test. Hatching rate analysis was done using the chi-square test. $P<0.05$ and $P<0.01$ were considered to be statistically significant $v s$ control.

\section{Results}

\section{Transcription profiles of HIPrx2}

The mRNA levels of HlPrx2 in whole female ticks and internal organs during blood-feeding and in different developmental stages (egg, larval, nymphal and adult stages) were investigated using real-time PCR. HlPrx2 mRNA was upregulated in whole female ticks, developmental stages, and all internal organs (salivary glands, midgut, ovaries, fat bodies, synganglia and hemocytes) during blood-feeding (Fig. 1). In the whole body, mRNA was upregulated at day 1 and, in spite of higher expression levels as compared to those of unfed stage (Welch's $t$-test: $\left.t_{(2)}=23.43, P=0.002\right)$, gradually decreased thereafter (Fig. 1a). Upregulation of the mRNA level was also observed in the developmental stages from unfed to engorgement, and the immature stages, including the egg, showed higher expression levels as compared to the
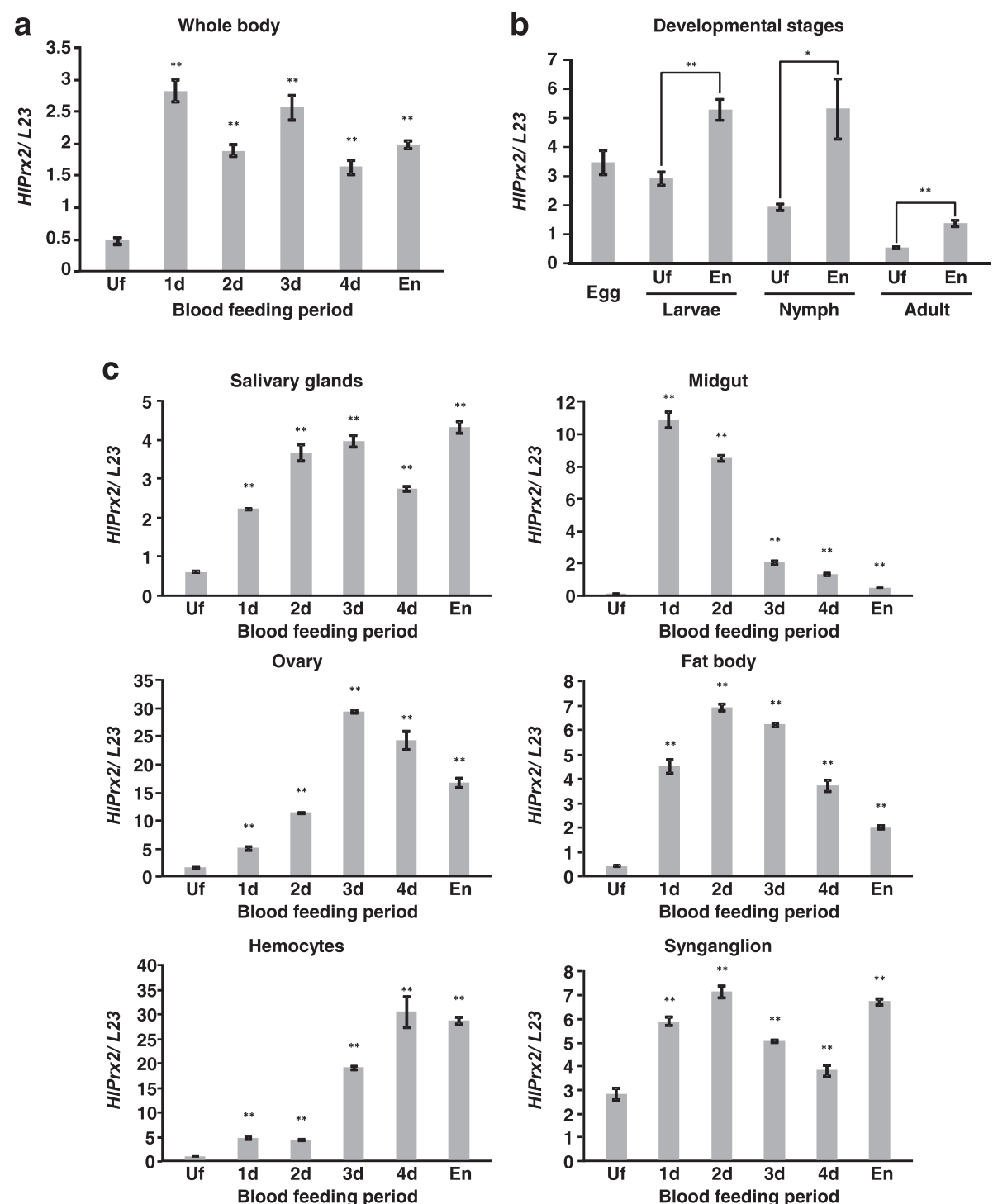

Fig. 1 a Transcription profiles of HIPrx2 in whole ticks during blood-feeding analyzed by real-time PCR (Uf, unfed females; 1d-4d, adults partially fed for 1-4 days). b Transcription profiles of HIPrx2 in unfed and engorged tick developmental stages. c Transcription profiles of HIPrx2 in the internal organs: salivary glands, midgut, ovary, fat body, hemocytes, synganglion). L23 was used as the internal control. Data are presented as the mean \pm standard deviation (SD). ${ }^{*} P<0.05 ;{ }^{* *} P<0.01$, significant differences vs dsLuc by Welch's $t$-test. Abbreviations: Uf, unfed ticks; En, engorged ticks 
adult stage (Welch's $t$-test: Larvae, $t_{(2)}=9.77, P=0.002$; Nymph, $t_{(2)}=5.65, \quad P=0.030 ;$ Adult, $t_{(2)}=12.39, \quad P=$ 0.006) (Fig. 1b). In the midgut, mRNA drastically increased at day 1 (Welch's $t$-test: $t_{(2)}=36.31, P=0.001$ ) and decreased thereafter (Fig. 1c, Midgut). In the ovary, the expression level gradually increased until day 2, then drastically increased at day 3 , and decreased thereafter (Fig. 1c, Ovary) (Welch's $t$-test: day $2, t_{(2)}=81.42, P<$ 0.001 ; day $\left.3, t_{(2)}=174.42, P<0.001\right)$. In the hemocytes, the expression level increased at day 1 and remained almost the same at day 2 , drastically increased from day 3 to day 4 (Welch's $t$-test: day $1, t_{(2)}=34.28, P=0.001$; day $2, t_{(2)}=40.81, P=0.001$; day $3, t_{(2)}=86.14, P<0.001$; day $\left.4, t_{(2)}=16.25, P=0.004\right)$, and then slightly decreased at the engorged state (Fig. 1c, Hemocytes). The expression levels of HlPrx2 gene in ovaries and hemocytes were higher than those of other internal organs. In other tissues, such as the salivary glands, fat bodies and synganglia, mRNA was upregulated from unfed to day 1 and remained at a high level until engorgement (Fig. 1c). These results indicate that the mRNA of HlPrx2 gene was upregulated in ticks by blood-feeding. The high levels of mRNA expression in the ovaries and hemocytes suggest that $H l P r x 2$ gene may be related to the reproduction and immune response of ticks.

\section{Protein expression profiles of HIPrx2}

The protein expression levels of HlPrx2 in whole female ticks and internal organs during blood-feeding and in different developmental stages were investigated by Western blot analysis using HIPrx2-specific antisera. The predicted molecular mass of HlPrx2 protein is approximately $22 \mathrm{kDa}$, and the theoretical isoelectric point (pI) is 6.8; the signal peptide and glycosylation sites were not found with sequence analysis [13]. However, the calculated molecular mass in Western blot analysis was approximately $26 \mathrm{kDa}$. The mobility of native HIPrx2 protein in Western blot analysis decreased because the $\mathrm{pI}=6.8$ is slightly low. HIPrx2 expression was generally upregulated during blood-feeding in the whole body, the developmental stages, and the midgut (Fig. 2). In the whole body and the developmental stages, the HlPrx2 expression level was upregulated from unfed to engorgement (Fig. 2a, b). Notably, in the developmental stages, protein expression levels seemed to be almost the same, although immature stages, including the egg, showed higher mRNA expression levels as compared to those of the adult stage (Figs. 1b and 2b). In Fig. 2b, other bands under HIPrx2 band at the engorged state of all stages can be seen. These bands are considered to be nonspecific bands derived from the blood of the host rabbit (see Additional file 1: Figure S1). These non-specific bands in the rabbit blood cross-reacted with HlPrx2 antisera; thus, these are considered to be a candidate cross-reacting protein related to 2-Cys peroxiredoxin (see Additional file 1: Figure S1 and Additional file 2). Moreover, in the knockdown ticks, the band of HlPrx2 protein was decreased as compared to the control group (see Additional file 1: Figure S2). Therefore, the antiHIPrx2 mouse serum used in this study was considered as specifically working.

In the midgut, although the protein expression level was very low in the unfed stage, it significantly increased from unfed to partially fed states (Welch's $t$-test: $t_{(2)}=$ 3.66, $P=0.049$ ) and significantly decreased in the engorged state (Welch's $t$-test: $t_{(2)}=3.34, P=0.034$ ) (Fig. 2c, Midgut). In the salivary glands, ovaries, and fat bodies, the protein expression levels of HlPrx2 were constant during blood-feeding (Fig. 2c). These results indicate that the protein expression of HIPrx2 is strongly upregulated in the whole body, especially in the midgut, by blood-feeding; however, the expression levels of HIPrx2 protein in the other tissues, such as the salivary glands, ovaries and fat bodies, were constant during blood-feeding. The drastic increase of HlPrx2 protein expression in the midgut during blood-feeding suggests that HIPrx2 protein could be related to the antioxidant response in this tissue because ticks' midgut may be exposed to high concentrations of ROS during blood-feeding.

\section{Localization of HIPrx2 in the salivary glands, midgut,} ovaries, and hemocytes from engorged adult female ticks Western blot analysis showed the high expression of HIPrx 2 protein in the whole body and internal organs. To determine localization in the cells of internal organs, IFAT was performed using some internal organs of engorged female ticks. In the salivary glands, positive fluorescence was detected in the cell membrane of the acinar cells (SA) and granular cells (SGG) and in the basal lamina of the salivary duct (SD) (Fig. 3, Salivary glands). In the midgut, positive fluorescence was detected in the basal lamina of the digestive cells (Fig. 3, Midgut). In the ovary, positive fluorescence was detected in the cell membrane of the oocytes and basal lamina of the oviduct (Fig. 3, Ovary), whereas in the hemocytes, positive fluorescence was detected in the cell membrane (Fig. 3, Hemocytes). These results demonstrate that the HlPrx2 protein was associated to the tissue membranes.

\section{Effects of HIPrx and/or HIPrx2 gene silencing on the blood-feeding and reproduction of female ticks}

To clarify the functions of the HlPrx and HlPrx2 genes, gene silencing using the RNAi method was conducted. Gene silencing was confirmed by semiquantitative RT-PCR and Western blot analysis (Fig. 4a). The knockdown of HlPrx and/or HlPrx2 caused significant differences in the ticks' engorged body weight, egg weight and hatching rate (Table 2). 

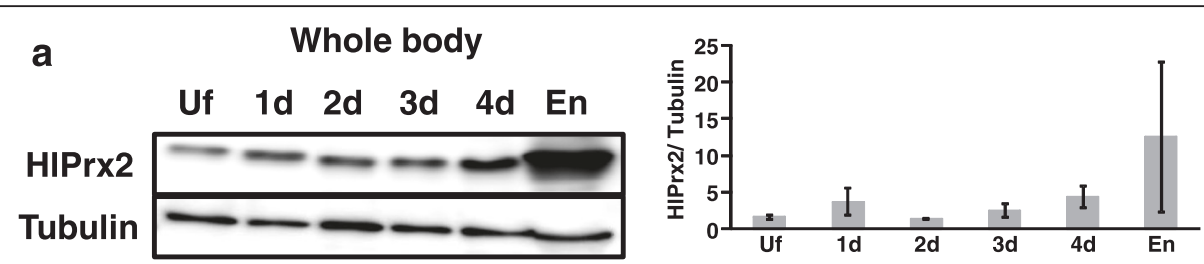

b

Developmental stages
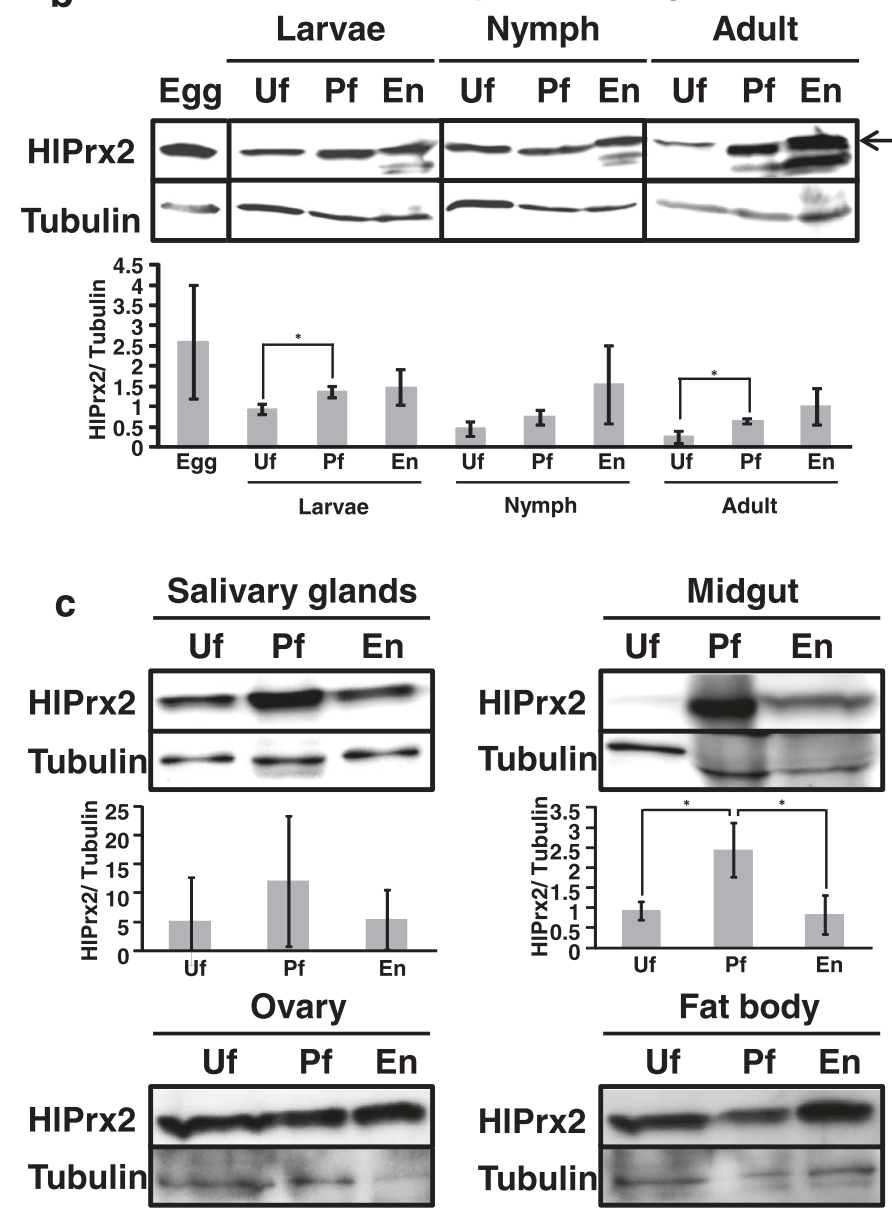

Fat body

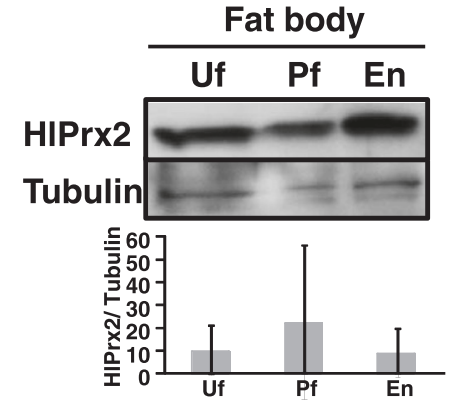

Fig. 2 a Protein expression profiles of HIPrx2 in whole ticks during blood-feeding as analyzed by Western blot analysis. b Protein expression profiles of HIPrx2 in developmental stages. Arrow indicates native HIPrx2 protein as distinguished from the non-specific bands below. c Protein expression profiles of HIPrx2 in the internal organs (salivary glands, midgut, ovary, fat body, hemocytes, and synganglion). For loading control, tubulin was detected. The bars show the results of band densitometry analysis for HIPrx2. The relative expression was calculated based on tubulin. Data are presented as the mean \pm standard deviation (SD). ${ }^{*} P<0.05$, significant differences by Welch's $t$-test. Abbreviations: Uf, unfed adults; Pf, partially fed adults at day 3 ; En, engorged adults

The ticks' engorged body weight and egg weight significantly decreased (Fig. 4b and c). Notably, double knockdowns, wherein both $H l P r x$ and $H l P r x 2$ were silenced, showed almost the same results as HlPrx silencing. HlPrx2 silencing resulted in a greater decrease in engorged body weight and egg weight when compared to those of dsDouble and dsHlPrx silencing. However, the hatching rates of $d s H l P r x$ and $d s H l$ Prx 2 groups were similar (Table 2). These results suggest that the knockdown of HlPrx and/or HlPrx2 

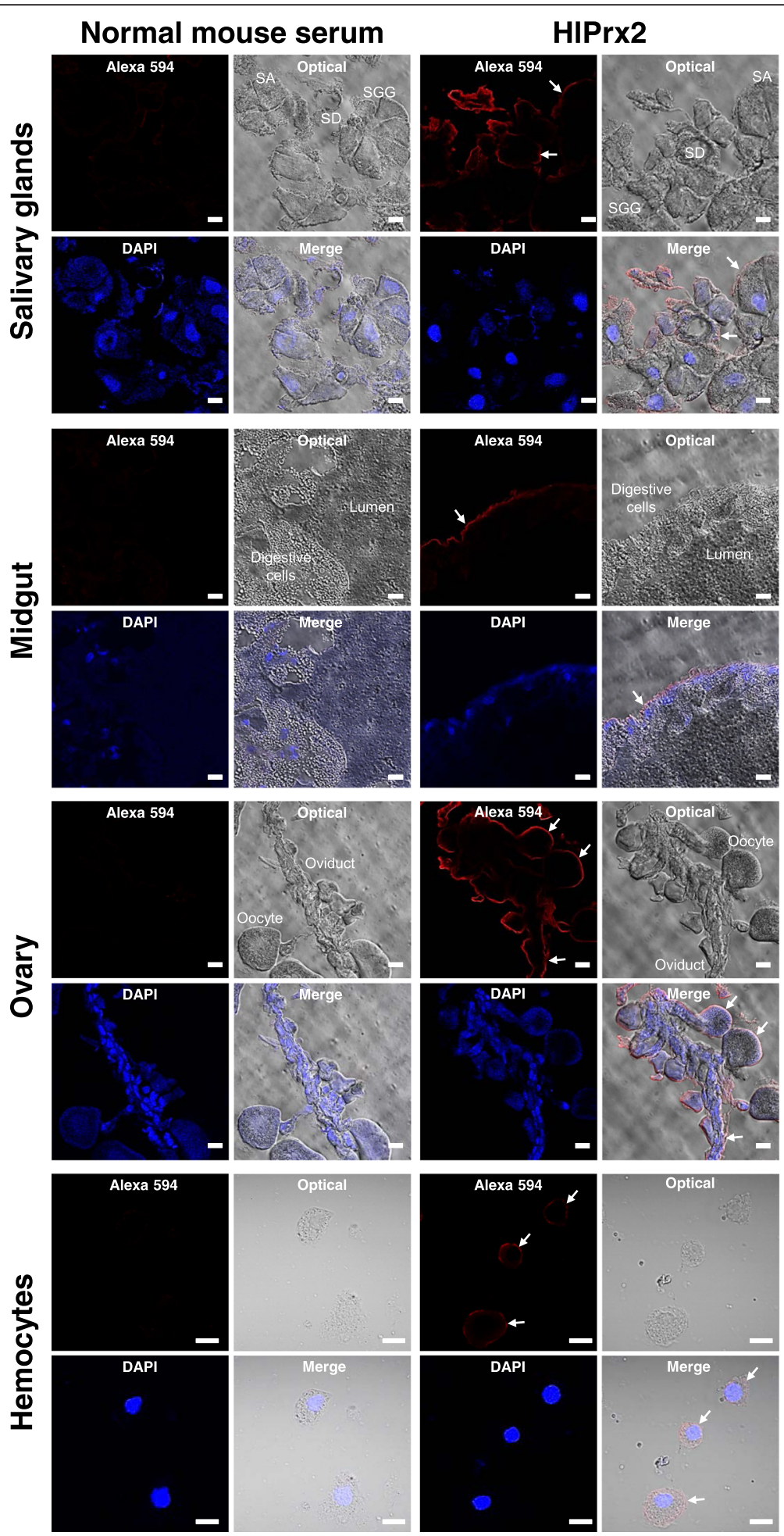

Fig. 3 Localization of HIPrx2 proteins in the salivary glands, midgut, ovary, and hemocytes from engorged adult ticks using IFAT under a confocal laser scanning microscope. Anti-HIPrx2 mouse serum was used as a primary antibody. Anti-mouse lgG conjugated with Alexa Fluor 594 was used as a secondary antibody and nuclei were visualized using DAPI. Normal mouse serum was used for control. Arrows indicate the specific fluorescence. Abbreviations: SA, salivary gland acinar cells; SGG, salivary gland granular cells; SD, salivary duct. Scale-bars: $20 \mu \mathrm{m}$ (salivary glands, midgut, and ovary) and $10 \mu \mathrm{m}$ (hemocytes) 


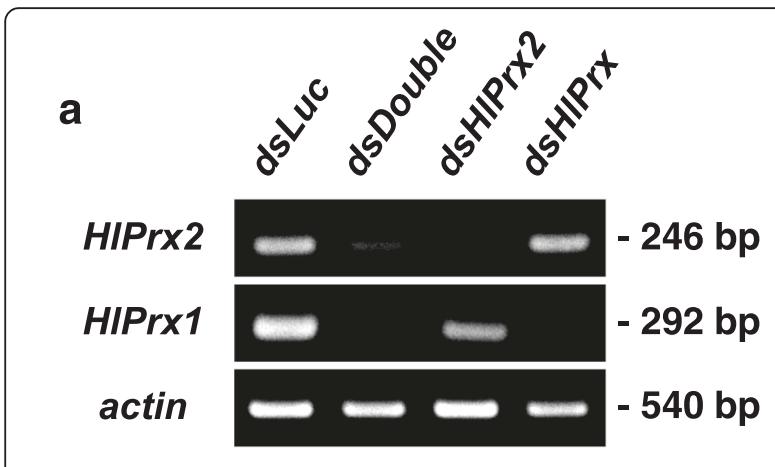

b

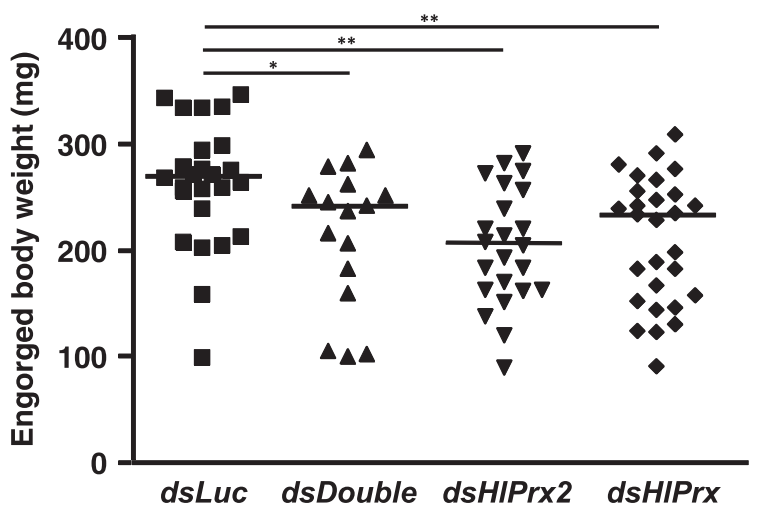

C

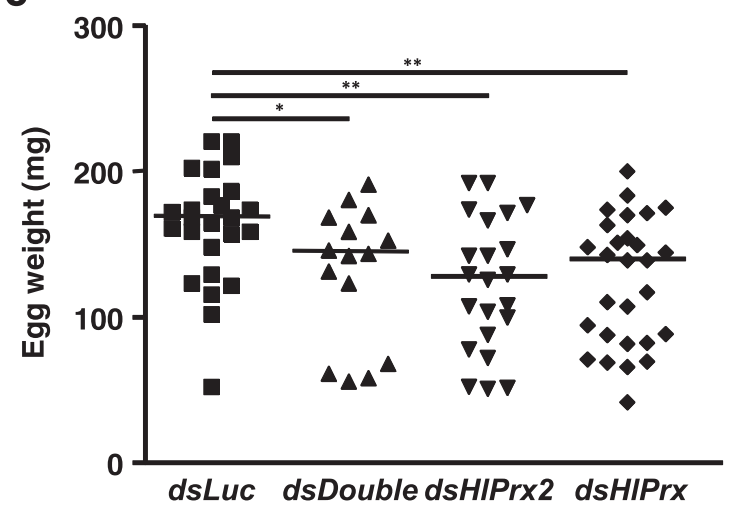

Fig. 4 a Knockdown confirmation of HIPrx and/or HIPrx2 genes in partially fed adult ticks. Each tick total RNA was extracted from 3 ticks pooled. The left column indicates the detection primer set; actin was used as a control. The right column indicates the size of the PCR products. $\mathbf{b}$ Column graph for engorged body weight in the knockdown experiment. c Column graph for egg weight after finishing oviposition by engorged adult ticks in the knockdown experiment. Horizontal lines indicate the median values. Abbreviations: dsLuc, double-stranded Luciferase-injected group; dsHIPrx2, doublestranded HIPrx2-injected group; dsHIPrx, double-stranded HIPrx-injected group; dsDouble, both double-stranded HIPrx- and HIPrx2-injected group. ${ }^{*} P<0.05 ;{ }^{* *} P<0.01$, significant differences vs dsLuc by Welch's $t$-test

genes significantly decreased engorged body weight, egg weight and hatching rate as compared to the dsLuc group.
Double knockdown of HIPrx genes increased the concentration of $\mathrm{H}_{2} \mathrm{O}_{2}$ before and after blood-feeding To elucidate the observed effects of HlPrx and/or $H l P r x 2$ gene silencing during blood-feeding, $\mathrm{H}_{2} \mathrm{O}_{2}$ concentrations were measured in female ticks. Gene silencing was also confirmed by semi-quantitative RT-PCR (data not shown). In the unfed and engorged states, the dsDouble group showed significantly higher concentrations of $\mathrm{H}_{2} \mathrm{O}_{2}$ as compared to the dsLuc group (Welch's $t$-test: Unfed, $t_{(10)}=7.77, P<0.001$; Engorged, $t_{(17)}=2.72$, $P=0.014$ ) (Fig. 5, Unfed and Engorged). HlPrx or HlPrx2 gene-silenced groups only showed slightly higher concentrations of $\mathrm{H}_{2} \mathrm{O}_{2}$ as compared to the dsLuc-injected group in the unfed state (Fig. 5, Unfed). On the other hand, in the engorged state, the HlPrx2 gene-silenced group showed a slightly higher concentration of $\mathrm{H}_{2} \mathrm{O}_{2}$, whereas the HlPrx gene-silenced group showed a slightly lower concentration of $\mathrm{H}_{2} \mathrm{O}_{2}$ as compared to the dsLucinjected group (Fig. 5, Engorged). These results demonstrate that the knockdown of HlPrx and HlPrx2 genes leads to a high concentration of $\mathrm{H}_{2} \mathrm{O}_{2}$ in ticks before and after blood-feeding.

\section{Discussion}

To protect against the toxicity of $\mathrm{H}_{2} \mathrm{O}_{2}$, aerobic organisms have evolved antioxidant enzymes, such as catalases, peroxidases, and Prxs [2]. Moreover, ticks lack heme synthesis and catabolism pathways because they are unable to prepare $\sigma$-aminolevulinic acid, a heme precursor, even at genomic levels [3, 19]. Therefore, they rely on heme from their host and store it in hemosomes of the midgut without digestion [4]. These facts suggest that ticks might face difficulties in producing proteins that contain heme, such as catalase and peroxidase, which are both $\mathrm{H}_{2} \mathrm{O}_{2}$-scavenging enzymes [20]. Moreover, ticks must acquire nutrients from the host blood meal and metabolize these nutrients via catabolism and anabolism [21]. Plasmodium parasites also take in nourishment from host blood and are likely to utilize members of the Prx family as the principal enzymes for reducing peroxides, including $\mathrm{H}_{2} \mathrm{O}_{2}$, because they lack catalase and peroxidase [22]. Therefore, Prxs might be similarly essential to the regulation of the $\mathrm{H}_{2} \mathrm{O}_{2}$ concentration for ticks.

In this study, we found that HlPrx 2 mRNA expression was upregulated by blood-feeding (Fig. 1). On the other hand, HIPrx 2 protein expression was almost stable during blood-feeding, except in the midgut (Fig. 2). In the whole body, although mRNA expression was upregulated by blood-feeding when compared to the unfed state (Fig. 1a, b), protein expression seemed to be constant in all states of blood-feeding except for the engorged state, where it showed an increased expression level (Fig. 2a, b). Fasciola parasites secrete Prxs into 
Table 2 Effects of HIPrx and/or HIPrx2 gene silencing in ticks

\begin{tabular}{|c|c|c|c|c|c|c|}
\hline Knockdown groups & Infest No. & Drop No. & $\begin{array}{l}\text { Engorged body } \\
\text { weight (mg) }\end{array}$ & Egg weight (mg) & Ratio of egg weight/engorged body weight & Hatching rate (\%) \\
\hline dsLuc & 30 & 25 & $263.7 \pm 58.9$ & $162.1 \pm 38.9$ & $0.61 \pm 0.03$ & 100 \\
\hline dsDouble & 30 & 15 & $218.8 \pm 66.2^{\mathrm{a}}$ & $130.3 \pm 46.7^{d}$ & $0.59 \pm 0.08$ & 87 \\
\hline ds HIPrx2 & 30 & 22 & $204.4 \pm 56.3^{b}$ & $116.7 \pm 45.3^{\mathrm{e}}$ & $0.55 \pm 0.09^{9}$ & $77^{i}$ \\
\hline$d s H I P r x$ & 30 & 28 & $210.0 \pm 59.8^{c}$ & $124.8 \pm 43.4^{f}$ & $0.58 \pm 0.06^{\mathrm{h}}$ & $78^{j}$ \\
\hline
\end{tabular}

${ }^{a}$ Significant difference as compared with the $d s L u c$ group by Welch's $t$-test: $t_{(27)}=3.16, P=0.020$

${ }^{\mathrm{b}}$ Significant difference as compared with the $d s L u c$ group by Welch's $t$-test: $t_{(45)}=3.53, P=0.001$

'Significant difference as compared with the $d s L u c$ group by Welch's $t$-test: $t_{(50)}=3.24, P=0.002$

${ }^{\mathrm{d}}$ Significant difference as compared with the $d s L u c$ group by Welch's $t$-test: $t_{(26)}=2.22, P=0.036$

'Significant difference as compared with the $d s L u c$ group by Welch's $t$-test: $t_{(42)}=3.67, P=0.001$

fSignificant difference as compared with the $d s L u c$ group by Welch's $t$-test: $t_{(51)}=3.30, P=0.002$

${ }^{9}$ Significant difference as compared with the $d s L u c$ group by Welch's $t$-test: $t_{(25)}=2.85, P=0.009$

${ }^{h}$ Significant difference as compared with the $d s L u c$ group by Welch's $t$-test: $t_{(41)}=2.46, P=0.018$

'Significant difference as compared with the $d s L u c$ group by Chi-square test: $X^{2}=6.36, d f=1, P=0.012$

jSignificant difference as compared with the $d s L u c$ group by Chi-square test: $X^{2}=6.04, d f=1, P=0.014$

their hosts to regulate their environment for survival in the host body [9]. Our results suggest that ticks may also secrete HlPrx2 protein into hosts as Fasciola parasites do, and the inconsistency of protein expression in comparison with mRNA expression may be due to the release of HlPrx2 proteins. Protein expression in the whole body increased according to the state of engorgement (Fig. 2c). This drastic change seems to be related to body size, because tick body weight notably increases from day 4 to engorgement, and the increase in body weight is about 100 -fold compared to unfed ticks [23]. It may be also in response to the very large amounts of blood ingested during the rapid engorgement stage, which may expose ticks to higher levels of ROS. Although other developmental stages (larval and nymphal stages) also showed similar tendencies in HIPrx2 protein expression (Fig. 2b), HlPrx2 mRNA expression in larval and nymphal stages was higher than in the adult stage (Fig. 1b). This result suggests that HlPrx2 protein might have an important role in the molting and survival of immature stages during blood-feeding and after engorgement.

In the internal organs, especially the midgut, HIPrx 2 mRNA and protein expression was consistent (Figs. 1c and 2c). The mRNA and protein expression levels were negligible in the unfed midgut (Figs. 1c and 2c). Blood-feeding acts as a trigger, inducing the upregulation of HIPrx2 mRNA and protein expression. In IFAT examination of the midgut, HIPrx2specific fluorescence was detected in the basal lamina (Fig. 3). There have been some reports that the multimer of 2-Cys Prxs are associated with membranes, such as red blood cells [24, 25]. Our results, along with those of previous reports, suggest that HlPrx2 protects digestive cells against membrane oxidation and suppresses unnecessary diffusion of $\mathrm{H}_{2} \mathrm{O}_{2}$ from midgut lumen and digestive cells. On the other hand, the midgut, ovaries, and fat bodies are known to produce vitellogenin, a phospholipoglycoprotein and a member of the lipid transfer protein superfamily that is the precursor of major yolk proteins in all oviparous organisms [26, 27]. During blood-feeding, the expression patterns of tick vitellogenin are upregulated from day 3 to engorgement; the highest expression of mRNA and protein is observed upon engorgement [27]. Vitellogenin also has a positive effect on oxidative stress resistance in bees and is a preferred target of oxidative carbonylation in comparison with hemolymph proteins in adult bees [28]. In addition, in the ovaries and fat bodies, HIPrx 2 mRNA expression was upregulated from around day 3 , and protein expression was present stably (Figs. 1c and 2c). This indicates HlPrx2 protein could protect vitellogenin and the organs synthesizing vitellogenin, such as the midgut, the fat bodies, and the ovaries, from the oxidative stress that occurs during blood-feeding. In the salivary glands, HlPrx2 mRNA expression was upregulated during blood-feeding (Fig. 1c), while protein expression was upregulated from unfed to partially fed states (Fig. 2c). Moreover, in the case of HlPrx, the other known peroxiredoxin of $H$. longicornis, mRNA is upregulated in the salivary glands, and HIPrx protein is also highly expressed in the salivary glands [12]. An anti-HlPrx antibody was detected in the host serum after several repeated tick infestations [12], suggesting that the HIPrx was released from ticks into the host eliciting to produce anti-HIPrx on immune response. In Fasciola parasites, infective parasites excyst from a dormant state following ingestion and penetrate the intestinal wall before migrating to the liver; in this nutrient- and oxygen-rich environment, the parasites undergo rapid growth and development, and energy is supplied by aerobic respiration [29]. This developmental situation of Fasciola parasites is similar to the 


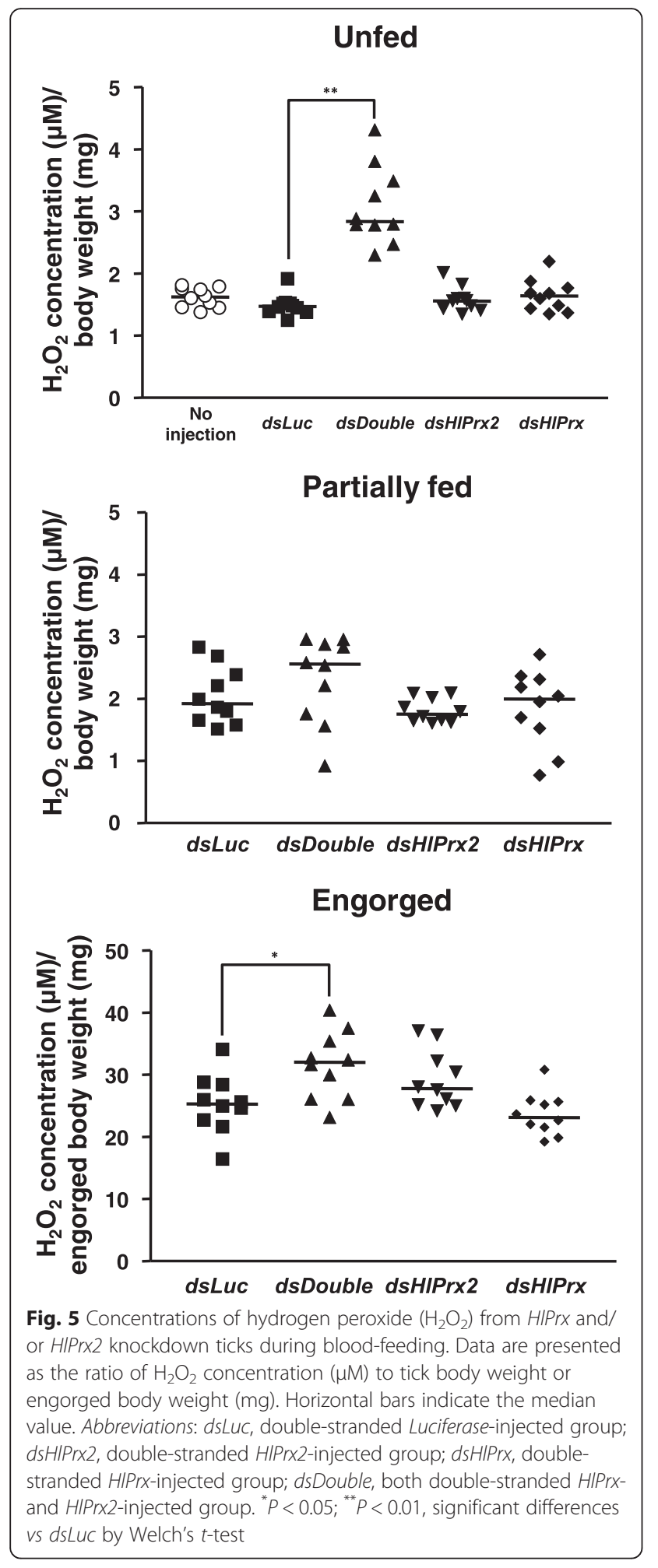

development of ticks during blood-feeding. In addition, Fasciola parasites secrete Prxs into their host to regulate their environment for survival in the host body [9]. These findings strongly suggest that tick Prxs may be also secreted into the host's body in a way similar to that of Fasciola parasites.

In hemocytes, $H l P r x 2$ mRNA expression was upregulated during blood-feeding, and a specific fluorescence was also detected in cell membranes of the hemocytes (Figs. 1c, 3). In Ixodes ricinus, two Prx homologous genes (Accession nos. AY333958 and AY333959) were strongly induced in the hemolymph after Borrelia burgdorferi infection [30]. Furthermore, Borrelia exploits the salivary Salp25D, a protein homologous to Prx in Ixodes scapularis, for protection against reactive oxygen intermediates generated by the mammalian neutrophils at the vector-host interface [31]. These results indicate that HIPrx2 might be related to immune response, e.g. digestion of foreign bodies such as Borrelia and Babesia parasites in hemocytes. In the mosquito Anopheles stephensi, 2-Cys Prx (AsPrx-4783) expression induced in the midgut was two to seven times higher in malaria parasiteinfected insects than in uninfected mosquitoes [32]. Two $\operatorname{Prx}$ genes of $I$. ricinus were also induced in the midgut by $B$. burgdorferi infection [30]. HlPrx2 in the midgut may also be involved in immune response; however, further investigation is necessary.

We also performed knockdown experiments of HlPrx and/or HlPrx2 genes and measured the $\mathrm{H}_{2} \mathrm{O}_{2}$ after the knockdown of these genes (Table 2 and Figs. 4, 5). The $\mathrm{H}_{2} \mathrm{O}_{2}$ concentration of no injection group in the unfed state was about $3 \mu \mathrm{M}$ (data not shown). In comparison with insects, the $\mathrm{H}_{2} \mathrm{O}_{2}$ concentration in normal state silkworms was also reported at about $3 \mu \mathrm{M}$ [33]. These observation may suggest that at a normal state, tick and silkworm $\mathrm{H}_{2} \mathrm{O}_{2}$ concentrations might have the same range. Therefore, this detection method of $\mathrm{H}_{2} \mathrm{O}_{2}$ concentration was considered as functionally acceptable. In the unfed and engorged states, the dsDouble group showed significantly higher concentrations of $\mathrm{H}_{2} \mathrm{O}_{2}$ as compared to the $d s L u c$ group. These results suggest a synergistic regulation of $\mathrm{H}_{2} \mathrm{O}_{2}$ by HIPrx and HIPrx2. In addition, phenotype evaluation after the knockdown of $H l P r x$ and/or HlPrx2 demonstrated significant decreases in the engorged body weight, egg weight and hatching rate, particularly after $H l P r x 2$ knockdown. The antioxidant activities evaluated by a metal-catalyzed oxidation system seemed to be almost the same comparing 1-Cys Prx and 2-Cys Prx from the bumblebee Bombus ignites [34]. The donors of 1-Cys Prxs and 2-Cys Prxs are thiol and thioredoxin, respectively [7]. Thioredoxin is a major disulfide reductase system which can provide electrons to a large range of enzymes and is found to be critical for DNA synthesis and defense against oxidative stress [35]. Taken together, the 1-Cys and 2-Cys Prxs seemed to have almost the same antioxidant activity but their donors are different. These data indicate that 2-Cys Prx is more related to cell metabolism through the 
antioxidant activity because of its utilization of thioredoxin as donor, thus, HlPrx2 knockdown in the ticks led to the significant decrease in engorged body weight, egg weight, and hatching rate in spite of no significant effect to $\mathrm{H}_{2} \mathrm{O}_{2}$ concentrations in the knockdowned ticks. Therefore, these findings suggest that HlPrxs play an important role in successful blood-feeding and reproduction, with HlPrx 2 being apparently more significant. Additionally, the observed effects in the dsDouble group were milder than those of the $d s H l P r x 2$ group. $\mathrm{H}_{2} \mathrm{O}_{2}$ can activate signaling pathways to stimulate cell proliferation, differentiation and migration in multicellular organisms [36]. These results suggest that the $d s D o u b l e$ group, but not the $d s H l P r x 2$ group, was exposed to a high concentration of $\mathrm{H}_{2} \mathrm{O}_{2}$, leading to higher engorged body weight, egg weight and hatching rate as compared to the $d s H l P r x 2$ group.

In endoparasites, Prx has been shown to be the most important detoxifying enzyme for their survival $[8,9]$ making it a candidate for use in vaccine development and a therapeutic target in treating endoparasitic infectious diseases [30, 32]. In ticks, there have been a few reports on Prxs. However, anti-HlPrx antibody was detected in the host serum after several repeated tick infestations [12], suggesting that HlPrx was released from ticks into the host and the amount of released HlPrx protein was quite small since several infestations of ticks were done to detect the anti-HlPrx antibody. In addition, ticks ingest and concentrate large amounts of the hostderived blood [23], it can be suggested that the antiHIPrx antibody would be concentrated in tick's body. In the present study, anti-HlPrx2 antibody cross-reacted with some rabbit Prx from normal rabbit blood (see Additional file 1: Figure S1), giving some concerns whether HlPrx2 can be a good vaccine candidate. However, the knockdown of HlPrx and/or HlPrx2 genes significantly affected tick blood-feeding, reproduction and antioxidant activity (Table 2 and Figs. 4, 5). Therefore, tick Prx can be a potential target for tick control and provide further understanding of the oxidative stress coping mechanisms in ticks during blood-feeding.

\section{Conclusion}

In summary, we investigated mRNA and protein expression profiles of HlPrx2 and the localization of this protein in tick tissues. Real-time PCR showed that HlPrx2 gene expression in whole bodies and internal organs was significantly upregulated during blood-feeding. However, protein expression was constant throughout blood-feeding. Moreover, a knockdown experiment of HlPrx 2 was performed using RNAi to evaluate its function in ticks. The knockdown of the HlPrx 2 gene caused significant differences in body weight, egg weight and hatching rate in engorged ticks as compared to those of the control group. Finally, the detection of $\mathrm{H}_{2} \mathrm{O}_{2}$ after the double knockdown of HlPrxs in ticks showed that $\mathrm{H}_{2} \mathrm{O}_{2}$ concentration increased before and after bloodfeeding. Therefore, HIPrx2 can be considered important for successful blood-feeding and reproduction through the regulation of $\mathrm{H}_{2} \mathrm{O}_{2}$ concentrations in ticks during blood-feeding. This study contributes to the search for a candidate target for tick control and furthers understanding of the tick's oxidative stress coping mechanism during blood-feeding.

\section{Additional files}

Additional file 1: Figure S1. Comparison of normal rabbit blood and engorged-state samples in developmental stages using Western blot analysis. The top arrow indicates native HIPrx2 protein, the middle arrow indicates non-specific band 1 and the bottom arrow indicates non-specific band $2(\mathrm{M}$, marker). Figure S2. Confirmation of antibody's specificity in HIPrx and/or HIPrx2 genes-silencing partially fed adult ticks. Each tick's total protein was extracted from 3 ticks pooled. The left column indicates the specific anti-serum. For loading control, tubulin was detected. (PPTX $11304 \mathrm{~kb}$ )

Additional file 2: Candidates for non-specific bands from Japanese white rabbit blood in Western blot analysis. (DOCX $13 \mathrm{~kb}$ )

\section{Acknowledgements}

We are grateful to Dr. T. Masatani of the Transboundary Animal Diseases Research Center, Joint Faculty of Veterinary Medicine, Kagoshima University, for his helpful comments and suggestions on this work.

\section{Funding}

This work was supported by the Japan Society for the Promotion of Science (JSPS) KAKENHI Grant Numbers 25292173, 26660229, 16H05028, and 16 J08221, and Cooperative Research Grant (27-joint-11) of the National Research Center for Protozoan Diseases, Obihiro University of Agriculture and Veterinary Medicine. K. Kusakisako is supported by a Grant-in-Aid for JSPS fellows.

\section{Availability of data and materials}

The datasets supporting the conclusions of this article are included within the article and its additional files. The sequences of HIPrx, HIPrx2, actin, tubulin, $P O$, and $L 23$ are deposited in the GenBank database under the accession number AB038382 (HIPrx), LC049075 (HIPrx2), AY254898 (actin), AB642157 (tubulin), EU048401 (PO), and DQ849041 (L23).

\section{Authors' contributions}

$\mathrm{KK}, \mathrm{MM}$ and $\Pi$ designed the experiments. KK, EPH, HM, and MRT performed the experiments. KK, RLG, RUS, NT, MM, KF and TT analyzed the data. KK wrote the manuscript. All the authors checked and approved the final version of the manuscript to be published.

\section{Competing interests}

The authors declare that they have no competing interests.

\section{Consent for publication}

Not applicable.

\section{Ethics approval and consent to participate} Not applicable.

\section{Author details}

${ }^{1}$ Laboratory of Infectious Diseases, Joint Faculty of Veterinary Medicine, Kagoshima University, Korimoto, Kagoshima 890-0065, Japan. ${ }^{2}$ Department of Pathological and Preventive Veterinary Science, United Graduate School of Veterinary Science, Yamaguchi University, Yoshida, Yamaguchi 753-8515, Japan. ${ }^{3}$ Department of Veterinary Paraclinical Sciences, College of Veterinary Medicine, University of the Philippines Los Baños, Los Baños, Laguna 4031, Philippines. ${ }^{4}$ National Research Center for Protozoan Diseases, Obihiro University of Agriculture and Veterinary Medicine, Inada-cho, Obihiro, 
Hokkaido 080-8555, Japan. ${ }^{5}$ Department of Parasitology, Kitasato University School of Medicine, Minami, Sagamihara, Kanagawa 252-0374, Japan. ${ }^{6}$ National Agricultural and Food Research Organization, Tsukuba, Ibaraki 305-0856, Japan.

Received: 27 June 2016 Accepted: 11 August 2016

Published online: 19 August 2016

\section{References}

1. Robinson MW, Hutchinson AT, Dalton JP, Donnelly S. Peroxiredoxin: a central player in immune modulation. Parasite Immunol. 2010;32:305-13.

2. Rhee SG. $\mathrm{H}_{2} \mathrm{O}_{2}$, a necessary evil for cell signaling. Science. 2006;312:1882-3.

3. Hall A, Nelson K, Poole LB, Karplus PA. Structure-based insights into the catalytic power and conformational dexterity of peroxiredoxins. Antioxid Redox Signal. 2011;15:795-815.

4. Chae HZ, Kim HJ, Kang SW, Rhee SG. Characterization of three isoforms of mammalian peroxiredoxin that reduce peroxides in the presence of thioredoxin. Diabetes Res Clin Pract. 1999:45:101-12.

5. Lee TH, Kim SU, Yu SL, Kim SH, Park DS, Moon HB, et al. Peroxiredoxin II is essential for sustaining life span of erythrocytes in mice. Blood. 2003;101:5033-8.

6. Hofmann B, Hecht HJ, Flohé L. Peroxiredoxins. Biol Chem. 2002;383:347-64.

7. Choi HJ, Kang SW, Yang CH, Rhee SG, Ryu SE. Crystal structure of a novel human peroxidase enzyme at 2.0 A resolution. Nat Struct Biol. 1998:5:400-6.

8. Kawazu S, Komaki-Yasuda K, Oku H, Kano S. Peroxiredoxins in malaria parasites: parasitologic aspects. Parasitol Int. 2008;57:1-7.

9. Dalton JP, Robinson MW, Mulcahy G, O'Neill SM, Donnelly S. Immunomodulatory molecules of Fasciola hepatica: candidates for both vaccine and immunotherapeutic development. Vet Parasitol. 2013;195:272-85.

10. Grandjean O. Blood digestion in Ornithodorus moubata Murray sensu stricto Walton females (Ixodoidea: Argasidae) II. Modifications of midgut cells related to the digestive cycle and to the triggering action of mating. Ann Parasitol Hum Comp. 1983;58:493-514.

11. Citelli M, Lara FA, da Silva VIJ, Oliveira PL. Oxidative stress impairs heme detoxification in the midgut of the cattle tick, Rhipicephalus (Boophilus) microplus. Mol Biochem Parasitol. 2007;151:81-8.

12. Tsuji N, Kamio T, Isobe T, Fujisaki K. Molecular characterization of a peroxiredoxin from the hard tick Haemaphysalis longicornis. Insect Mol Biol. 2001:10:121-9

13. Kusakisako K, Masatani T, Miyata T, Galay RL, Maeda H, Talactac MR, et al. Functional analysis of recombinant 2-Cys peroxiredoxin from the hard tick Haemaphysalis longicornis. Insect Mol Biol. 2016;25:16-23.

14. Fujisaki K. Development of acquired resistance precipitating antibody in rabbits experimentally infested with females of Haemaphysalis longicornis (Ixodoidea: Ixodidae). Natl Inst Anim Health Q (Tokyo). 1978;18:27-38.

15. Umemiya-Shirafuji R, Tanaka T, Boldbaatar D, Tanaka T, Fujisaki K. Akt is an essential player in regulating cell/organ growth at the adult stage in the hard tick Haemaphysalis longicornis. Insect Biochem Mol Biol. 2012;42:164-73.

16. Galay RL, Takechi R, Umemiya-Shirafuji R, Talactac MR, Maeda $H$, Kusakisako K, et al. Impaired cellular immune response to injected bacteria after knockdown of ferritin genes in the hard tick Haemaphysalis longicornis. Parasitol Int. 2016;65:251-7.

17. Oba Y, Yoshida M, Shintani T, Furuhashi M, Inouye S. Firefly luciferase genes from the subfamilies Psilocladinae and Ototretinae (Lampyridae, Coleoptera). Comp Biochem Physiol B Biochem Mol Biol. 2012;161:110-6.

18. Low FM, Hampton MB, Peskin AV, Winterbourn CC. Peroxiredoxin 2 functions as a noncatalytic scavenger of low-level hydrogen peroxide in the erythrocyte. Blood. 2007;109:2611-7.

19. Perner J, Sobotka R, Sima R, Konvickova J, Sojka D, Oliveira PL, et al. Acquisition of exogenous haem is essential for tick reproduction. Elife. 2016:5:e12318.

20. Wood ZA, Schröder E, Robin Harris J, Poole LB. Structure, mechanism and regulation of peroxiredoxins. Trends Biochem Sci. 2003;28:32-40.

21. Tsuji N, Miyoshi T, Battsetseg B, Matsuo T, Xuan X, Fujisaki K. A cysteine protease is critical for Babesia spp. transmission in Haemaphysalis ticks. PLoS Pathog. 2008;4:e1000062.

22. Mitozo PA, de Souza LF, Loch-Neckel G, Flesch S, Maris AF, Fiqueiredo CP, et al. A study of the relative importance of the peroxiredoxin-, catalase-, and glutathione-dependent systems in neural peroxide metabolism. Free Radic Biol Med. 2011;51:69-77.
23. Kitaoka S. Physiological and ecological studies on some ticks. VII. Parthenogenetic and bisexual races of Haemaphysalis bispinosa in Japan and experimental crossing between them. Natl Inst Anim Health Q (Tokyo). 1961;1:142-9.

24. Schröder E, Littlechild JA, Lebedev AA, Errington N, Vagin AA, Isupov MN. Crystal structure of decameric 2-Cys peroxiredoxin from human erythrocytes at 1.7 A resolution. Structure. 2000;8:605-15.

25. Hall A, Karplus PA, Poole LB. Typical 2-Cys peroxiredoxins - structures, mechanisms and functions. FEBS J. 2009;276:2469-77.

26. Avarre JC, Lubzens E, Babin PJ. Apolipocrustacein, formerly vitellogenin, is the major egg yolk precursor protein in decapod crustaceans and is homologous to insect apolipophorin II/I and vertebrate apolipoprotein B. BMC Evol Biol. 2007;7:3.

27. Boldbaatar D, Umemiya-Shirafuji R, Liao M, Tanaka T, Xuan X, Fujisaki K. Multiple vitellogenins from the Haemaphysalis longicornis tick are crucial for ovarian development. J Insect Physiol. 2010;56:1587-98.

28. Seehuus SC, Norberg K, Gimsa U, Krekling T, Amdam GV. Reproductive protein protects functionally sterile honey bee workers from oxidative stress. Proc Natl Acad Sci USA. 2006;103:962-7.

29. Sekiya M, Mulcahy G, Irwin JA, Stack CM, Donnelly SM, Xu W, et al. Biochemical characterisation of the recombinant peroxiredoxin (FhePrx) of the liver fluke. Fasciola hepatica FEBS Lett. 2006:580:5016-22.

30. Rudenko N, Golovchenko M, Edwards MJ, Grubhoffer L. Differential expression of Ixodes ricinus tick genes induced by blood feeding or Borrelia burgdorferi infection. J Med Entomol. 2005;42:36-41.

31. Narasimhan S, Sukumaran B, Bozdogan U, Thomas V, Liang X, DePonte $K$, et al. A tick antioxidant facilitates the Lyme disease agent's successful migration from the mammalian host to the arthropod vector. Cell Host Microbe. 2007;2:7-18.

32. Peterson TM, Luckhart S. A mosquito 2-Cys peroxiredoxin protects against nitrosative and oxidative stresses associated with malaria parasite infection. Free Radic Biol Med. 2006;40:1067-82.

33. Zhang $L, L u Z$. Expression, purification and characterization of an atypical 2-Cys peroxiredoxin from the silkworm. Bombyx mori Insect Mol Biol. 2015;24:203-12.

34. Hu Z, Lee KS, Choo YM, Yoon HJ, Lee SM, Lee JH, et al. Molecular cloning and characterization of 1-Cys and 2-Cys peroxiredoxins from the bumblebee Bombus ignitus. Comp Biochem Physiol B Biochem Mol Biol. 2010;155:272-80.

35. Lu J, Holmgren A. The thioredoxin antioxidant system. Free Radic Biol Med. 2014:66:75-87.

36. Veal EA, Day AM, Morgan BA. Hydrogen peroxide sensing and signaling Mol Cell. 2007;26:1-14

\section{Submit your next manuscript to BioMed Central and we will help you at every step:}

- We accept pre-submission inquiries

- Our selector tool helps you to find the most relevant journal

- We provide round the clock customer support

- Convenient online submission

- Thorough peer review

- Inclusion in PubMed and all major indexing services

- Maximum visibility for your research

Submit your manuscript at www.biomedcentral.com/submit
Biomed Central 